\title{
The Retinal Posterior Pole in Early Parkinson's Disease: A Fundus Perimetry and SD-OCT Study
}

\author{
Massimo Cesareo' \\ Ernesto Di Marco ${ }^{1}{ }^{1}$ \\ Clarissa Giannini' \\ Matteo Di Marino (D) \\ Francesco Aiello' \\ Antonio Pisani ${ }^{2}$ \\ Mariangela Pierantozzi ${ }^{3}$ \\ Nicola Biagio Mercuri ${ }^{4,5}$ \\ Carlo Nuccil \\ Raffaele Mancino' \\ 'Ophthalmology Unit, Department of \\ Experimental Medicine, University of \\ Rome Tor Vergata, Rome, Italy; \\ ${ }^{2}$ Department of Brain and Behavioral \\ Sciences, University of Pavia, Pavia, Italy; \\ ${ }^{3}$ Parkinson's Center, Department of \\ Systems Medicine, University of Rome \\ Tor Vergata, Rome, Italy; ${ }^{4}$ Neurology, \\ Department of Systems Medicine, \\ University of Rome Tor Vergata, Rome, \\ Italy; ${ }^{5}$ Fondazione Santa Lucia I.R.C.C.S, \\ Rome, Italy
}

Correspondence: Massimo Cesareo; Ernesto Di Marco

Ophthalmology Unit, Department of Experimental Medicine University of Rome "Tor Vergata", via Montpellier, Rome, 100133, Italy

Tel/Fax +390620902973

Email massimo.cesareo@uniroma2.it; Ernesto260I@hotmail.it
Purpose: To assess the structure and function of the retinal posterior pole in patients with early Parkinson's disease (PD) and to identify possible biomarkers correlated with clinical features.

Patients and Methods: A cross-sectional case-control study of 21 patients with PD and 22 age-matched healthy controls (HC) was conducted. All subjects underwent full ophthalmological examinations, fundus perimetry (FP) and spectral domain-OCT (SD-OCT) of the entire retinal posterior pole and peripapillary retinal nerve fiber layer (pRNFL).

Results: We analyzed 41 eyes from 21 patients (14 males and 7 females) with early PD (Hoehn and Yahr scale (H\&Y) equal to or less than stage 2) and 41 eyes from $22 \mathrm{HC}$ (12 males and 10 females). We found no significant difference in the pRNFL thickness between patients with PD and HC. The statistical analysis of the SD-OCT posterior pole area, consisting of 64 values for each retinal layer, revealed a decrease in the outer nuclear layer (ONL) thickness in patients with PD $(\mathrm{p}<0.0001)$. On the contrary, a significant increase in the thickness of the outer plexiform layer (OPL) $(\mathrm{p}<0.0001)$ and of the retinal pigmented epithelium (RPE) $(\mathrm{p}=0.002)$ compared to healthy controls was detected. Other retinal layers showed no significant statistical differences. The differential light sensitivity (DLS) values measured by FP were significantly lower in patients than the healthy controls (15 [13-16.2] vs 17.95 [16.08-18.96] $\mathrm{p}<0.0001)$.

Conclusion: Our results showed that DLS and retinal structure differed in the posterior pole between patients with early PD and controls. Thickening of the OPL may represent accumulation of $\alpha$-synuclein in the OPL of patients with PD.

Keywords: Parkinson's disease, optical coherence tomography, retina, dopamine, alphasynuclein

\section{Introduction}

Parkinson's disease (PD) is the second most common neurodegenerative disorder, whose neuropathological stigmata are the loss of dopaminergic neurons in the Substantia Nigra pars compacta (SNpc) and the progressive accumulation of $\alpha$ synuclein ( $\alpha$-syn) into insoluble fibrillar inclusions known as Lewy bodies (LB). PD is a progressive and disabling disorder, including classical motor signs (bradykinesia, tremor, rigidity, and gait/postural disorders) and a heterogeneous cohort of nonmotor symptoms (eg neuropsychiatric, sensorial, autonomic, sleep disturbances). ${ }^{1,2}$

Despite important advances, some critical issues related to the disease remain to be addressed. Specifically, PD lacks either effective disease-modifying treatments or reliable biomarkers to diagnose and predict the clinical progression of the pathology. $^{3-5}$ 
Cerebrospinal Fluid (CSF) biomarkers, namely neurodegeneration-related proteins, such as tau proteins, $\alpha$-syn, and 42 -amyloid- $\beta$ peptide (A $\beta 42)$ allow the tracking of occurring pathological events. Notwithstanding the translational value and the clinical relevance, the use of CSF biomarkers is burdened by the invasiveness of CSF sampling, which frightens patients and limits repetitive assessment. ${ }^{1}$

On the other hand, innovative techniques of neuroimaging allow the non-invasive tracking of brain molecular targets, providing numerous biomarkers; however, either the scarce availability of these techniques or the expensive costs prevent the diffusion in the routine clinical practice. ${ }^{6}$

Patients with PD show a huge variety of visual, ocular and retinal changes related to disease pathology, which could be easily detected and measured by ophthalmological techniques. From this perspective, the eye can be considered an ideal site to assess the neuropathological PD modifications using accessible and non-invasive methodologies, thus representing a possible source of candidate biomarkers. ${ }^{7}$

In particular, the Spectral-domain Optical Coherence Tomography (SD-OCT) allows a non-invasive, repeatable and reproducible evaluation of the retinal structure by measuring the thickness of each retinal layer. Researchers demonstrated that the inner retinal layer thickness can be considered a biomarker associated with neurodegenerative diseases. $^{8}$

Fundus Perimetry (FP), or Microperimetry (MP), enables accurate retinotopic mapping of differential light sensitivity (DLS) and fixation. Both the SD-OCT and FP have been recently combined and used as a new method to superimpose the FP sensitivity map onto the SD-OCT volumetric map. The combination of the two techniques permits the exploration of the different retinal morphology and thickness at the posterior pole (PP) and the co-localization with differential light sensitivity values in patients with different retinal and/or neuro-ophthalmological diseases. ${ }^{9}$

Scientific evidence regarding the assessment of the retinal layers and the peripapillary nerve fiber layer in patients with PD remains controversial. Some studies found no significant difference between controls and patients with PD. ${ }^{10}$ Instead, other studies in which RNFL thickness, macular thickness, or foveal thickness were measured in the eyes of patients with PD reported a significant decrease in all these parameters when compared with healthy controls. ${ }^{11-14}$
In this study, FP and SD-OCT were used in colocalization $^{9}$ to assess the structure and function of the retinal posterior pole of the eyes of PD patients at an early stage, in order to identify possible ophthalmological biomarkers correlated with clinical features.

\section{Materials and Methods}

The study was approved by the independent ethical committee "Comitato Etico Indipendente del Policlinico Universitario Tor Vergata" and was in accordance with the ethical standards stated in the 1964 declaration of Helsinki. Informed consent was obtained from all participants.

In this study, we consecutively recruited patients admitted at the Clinics of Neurology and Ophthalmology of the University of Rome "Tor Vergata"; eligible patients had a diagnosis of idiopathic PD, according to Movement Disorder Society (MDS) Clinical Diagnostic Criteria. ${ }^{15}$

Data on disease onset, clinical characteristics, disease duration, dopaminergic treatment, and disease severity were collected.

Patients were also required to meet the following inclusion criteria: a disease diagnosis equal to or less than 4 years; a disease severity, as scored by Hoehn and Yahr scale (H\&Y), equal to or less than stage 2 in order to achieve an early PD group of patients, Mini-Mental Status Examination $($ MMSE) $>24$ (adjusted for age and educational level).

A group of healthy controls, without history or clinical signs of neurological diseases, homogeneous by age and sex with patients with PD was recruited among volunteers who came to our clinic for routine health checkup.

For both PD and HC subjects, exclusion criteria were a history of ocular trauma, previous ocular surgery that could impair retinal morphology and function, concurrent ocular diseases (including retina, optic nerve, cornea, and macular diseases), spherical refractive errors higher than 6 diopters, astigmatic errors greater than $\pm 2.00 \mathrm{D}$, use of drugs that could affect visual function, intraocular pressure (IOP) $>21 \mathrm{mmHg}$, systemic conditions that could impair the visual system (diabetes mellitus, uncontrolled hypertension or hypotension, cardiovascular diseases, renal failure), smoking habit and any other neurological disease.

Finally, patients and healthy controls were also excluded if their BCVA was worse than 0.0 LogMAR.

For each participant with PD, information on age, sex, duration of disease, Hoehn and Yahr (H\&Y) stage and Movement Disorder Society-Unified Parkinson's Disease 
Rating Scale Part III (MDS-UPDRS-III), levodopa equivalent daily dose (LEDD) were collected after neurological evaluation by an expert neurologist. Considering the observational nature of this study, patients on therapy maintained their regular treatment depending on their needs and current clinical practice.

Once neurological examination was completed, all patients underwent full ophthalmological examination including best-corrected visual acuity (BCVA), biomicroscopy evaluation with a slit lamp, and a Goldmann applanation tonometry.

\section{Optical Coherence Tomography}

Each patient, after the instillation of $1 \%$ phenylephrine + $0.5 \%$ tropicamide mydriatic eye drops (Visumidriatic Fenilefrina, Visufarma), underwent Spectralis SD-OCT examination; OCT scans were acquired using the TruTrack eye-tracking image alignment software (6.15.7.0, Heidelberg Engineering, Heidelberg, Germany). All Heidelberg Engineering imaging tools use Heidelberg Eye Explorer (HEYEX) database software installed on a dedicated computer. Image acquisition and analysis are also controlled by HEYEX software modules specific to each instrument.

The following scanning protocols of the "Glaucoma Module Premium Edition" software were used:

- "peripapillary RNFL" (pRNFL) scan that performs a circular peripapillary scan with a diameter of $3.5 \mathrm{~mm}$, which measures the thickness of the retina comprised within ILM and GCL, subdivided into the temporal (T), Supero-Temporal (ST), Supero-Nasal (ST), nasal (N), infero-nasal (IN), inferotemporal (IT), global (G) sectors.

- "Posterior Pole Vertical" scan that consists of volumetric measurements of the retinal posterior pole including an area of $30^{\circ} \times 25^{\circ}$ by means of 61 single vertical axial scans $120 \mu \mathrm{m}$ apart in the central 20, with a quadrangular geometry grid centred on the fovea, consisting of 64 volumetric units with an inclination fovea-todisc determined by the APS, with an ART equal to 15 .

Both of these scanning protocols are implemented with the APS (Anatomic Position System), which creates an anatomical map of each eye examined using two precise reference points: the center of the fovea and the center of the Bruch's membrane opening at the optic nerve head level. This allows a precise examination of the detected structures (Figure 1), ensures an accurate comparison with the reference data and high sensitive assessment of the structural change.

Poor quality scans, with a signal quality $<25$ or misalignments were rejected prior to automatic segmentation. Both HC and the PD images were reviewed to account for any segmentation algorithm failure.

\section{Microperimetry}

After pharmacological mydriasis and the OCT examination, patients and healthy controls underwent fundus perimetry with a MP1 Microperimeter (software version 1.7.3, Nidek Technologies Srl, Vigonza, PD, Italy) using a custom program called "PPole MP1" with 4-2 threshold strategy with pre-test, consisting of an examination grid pattern of 64 ( 8 horizontal x 8 vertical) test points and one foveal test point.

The grid is tilted as the "Posterior Pole" scan of the Spectralis SD-OCT [6] in order to overlap FP results onto the SD-OCT map. A 4-2 threshold strategy with pre-test was used to generate a random sequence of "Goldmann" III dimension stimuli on the MP1 LCD monitor; a single $3^{\circ}$ red cross was used as a fixation target. Before testing,

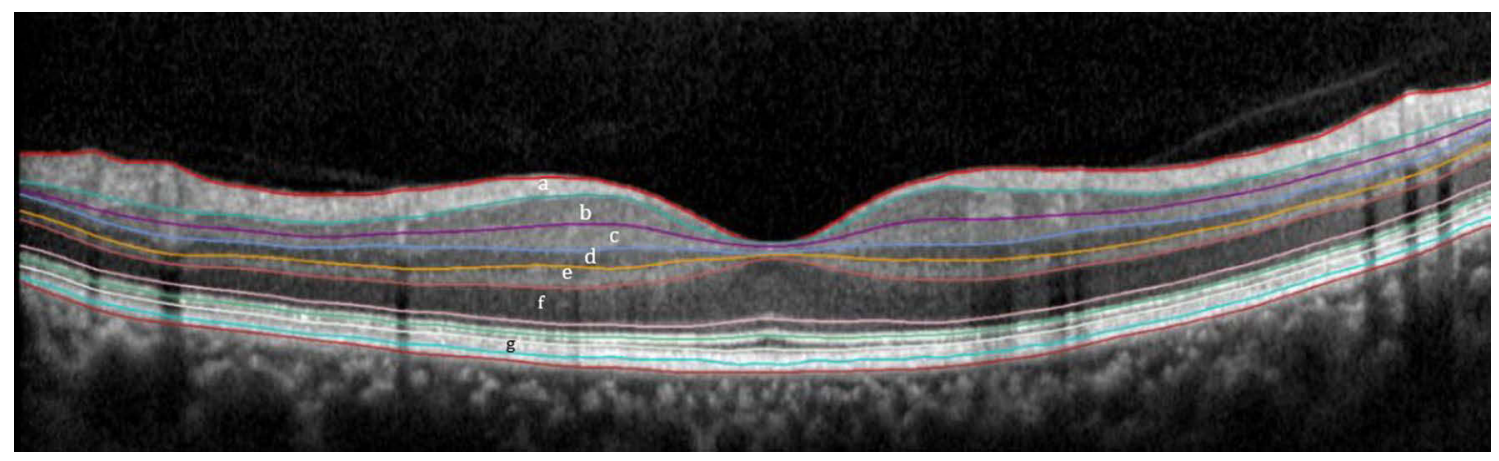

Figure I Retinal segmentation. Retinal segmentation as performed by the software for the SD-OCT (Spectralis): a - retinal nerve fiber layer (RNFL), $b$ - ganglion cell layer $(G C L)$, c - inner plexiform layer (IPL), d - inner nuclear layer (INL), e - outer plexiform layer (OPL), $f$ - outer nuclear layer (ONL) and $g$ - retinal pigment epithelium (RPE). 
all participants had to adapt to low light conditions during a five-minute period.

The MP1 microperimetry has an eye-tracking system that allows the detections of patient's eye movements by $25 \mathrm{Hertz}$ automatic recording of the fundus position with respect to the fixed retinal image acquired at the beginning of the exam. MP1 software allows an accurate topographic overlapping of the DLS results onto the previously obtained SD-OCT volumetric maps for each retinal layer ${ }^{9}$ (Figures 2 and 3).

\section{Statistical Analysis}

All data were initially entered into an EXCEL database (Microsoft, Redmond, Washington, United States). The analysis was performed using the SPSS software (SPSS 23.00 SPSS Inc., Chicago, IL). Descriptive statistics were calculated for all parameters.

Descriptive statistics consisted of the mean \pm standard deviation (SD) values for parameters with Gaussian distributions, after confirmation with histograms and the Kolmogorov-Smirnov test or median and range $\left(1^{\circ}\right.$ interquartile $-3^{\circ}$ interquartile) for variables with non-normal distributions.

Comparison among groups was performed by a one-way analysis of variance (ANOVA) for normal variables, MannWhitney test for non-parametric variables and the Chi-Square test for categorical variables. A P-value $<0.05$ was considered to be statistically significant.

Benjamini-Hochberg procedure for multiple hypothesis testing with a false discovery rate set to $10 \%$ was performed.

In particular, the ANOVA test was used to compare the following group parameters of patients with PD and healthy controls:

- The thickness of the pRNFL in the different sectors (T, TS, G, NS, N, NI, TI).

- The thickness of each of the $3^{\circ} \times 3^{\circ}$ volumetric units for each layer of the retinal Posterior Pole scan.

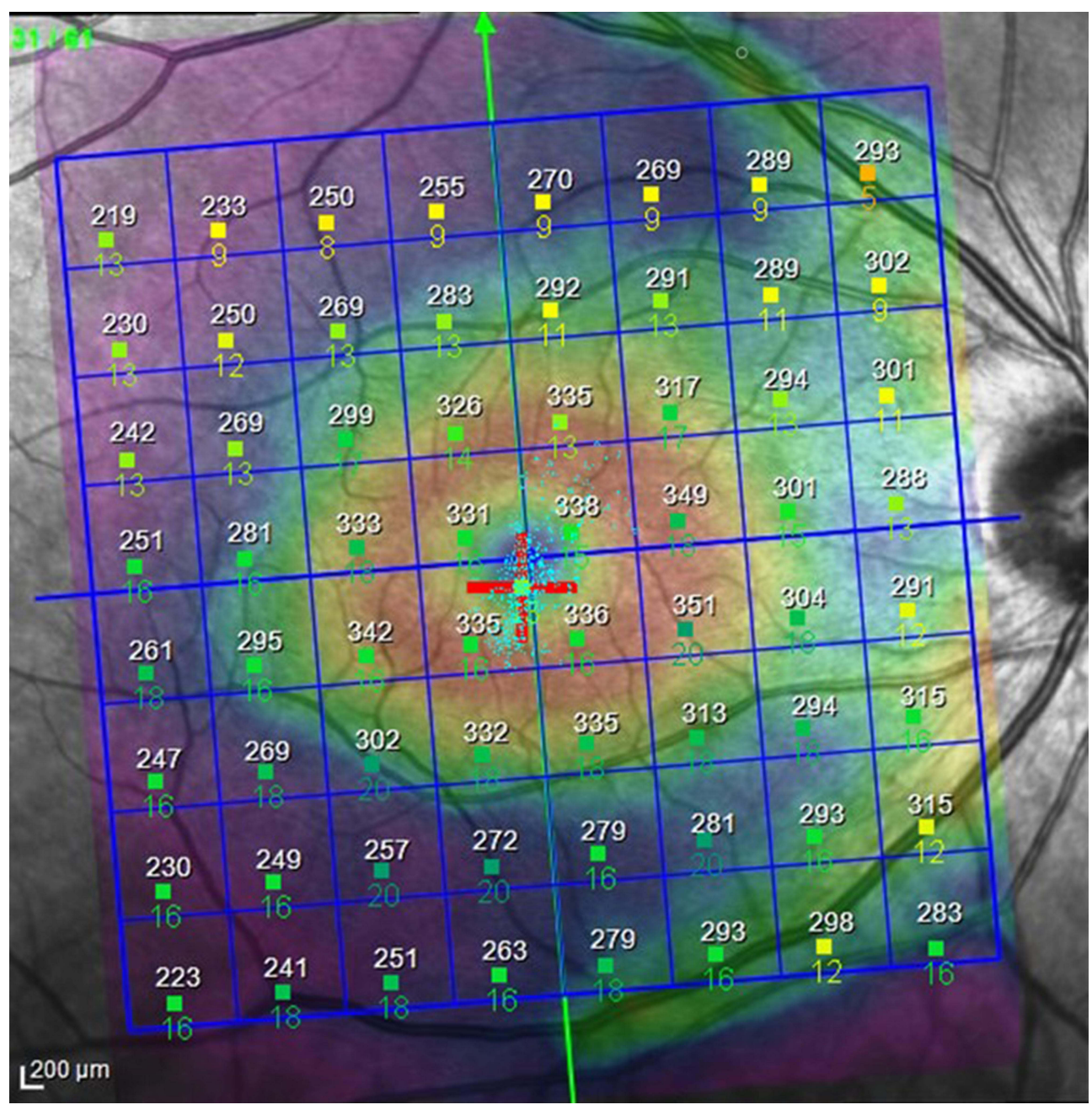

Figure 2 Combination map: MP DLS values are displayed in co-localization with SD-OCT "Posterior Pole" volumetric measures. 

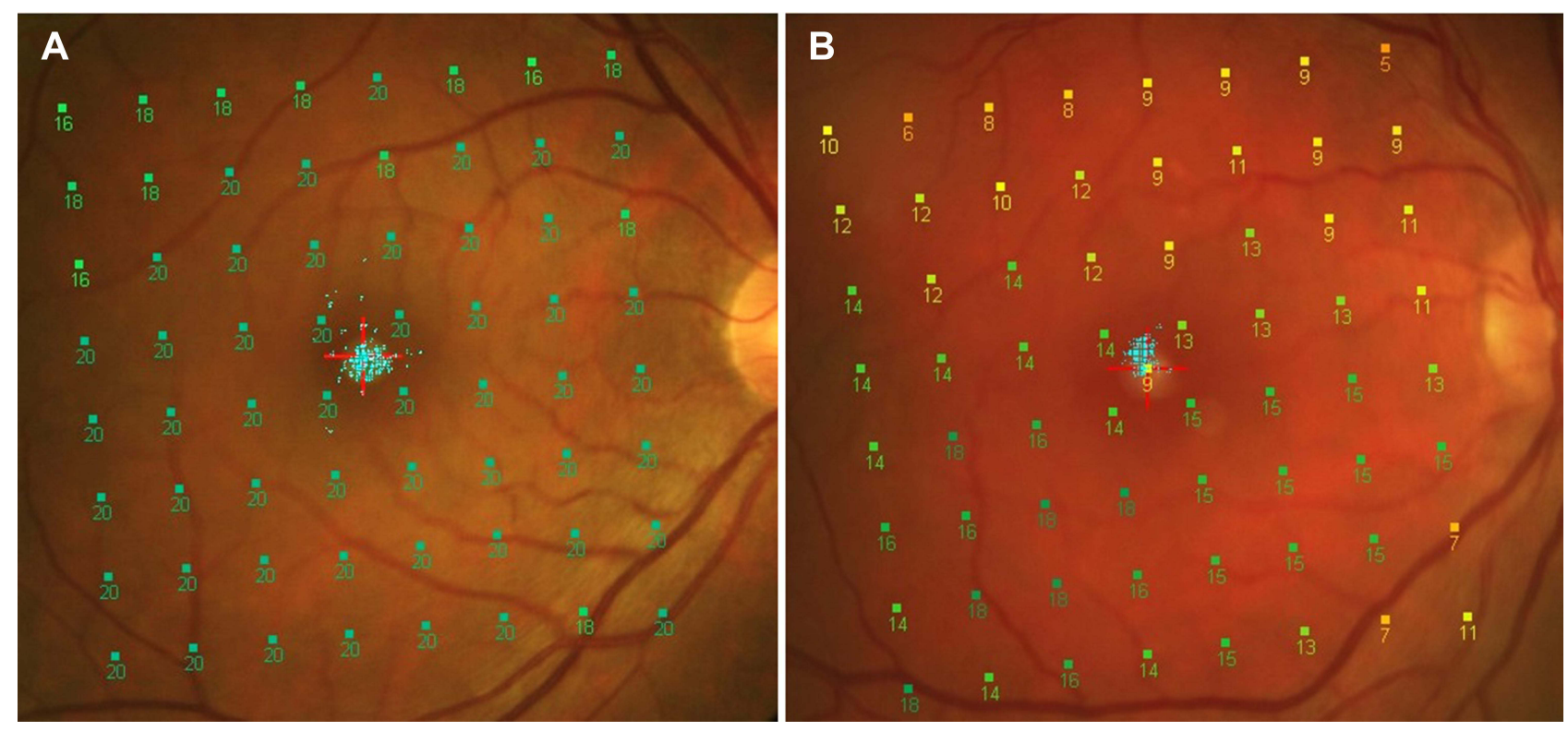

Figure 3 PPole MPI map of a healthy control and a patient with Parkinson's disease. (A) PPole MPI map showing normal values in the right eye of a HC. (B) PPole MPI map showing a decrease in DLS values throughout the whole pattern in the right eye of Patient with PD.

The Mann-Whitney test was used to compare the following group parameters of patients and healthy controls:

- The DLS values of the 64 test points of the retinal PP grid pattern and foveal DLS.

The correlation between the volumetric results detected by SD-OCT and the DLS values component measured by the MP1 microperimetry was carried out using the Pearson coefficient formula (r).

\section{Results}

We included 26 patients and $22 \mathrm{HC}, 4$ patients were excluded from the study based on medical history due to ocular pathology that could interfere with retina structure and 1 patient was excluded because of sub-optimal image quality. Individual eyes of 1 patient and $3 \mathrm{HC}$ were not included because the images were not obtained.

Therefore, we analyzed 41 eyes of 21 patients (14 males and 7 females) with PD and 41 eyes from 22 healthy controls (12 males and 10 females). The age range of patients recruited was 41-80 years (mean $63.10 \pm 10.32$ ) for parkinsonians and 46-80 (mean $57.89 \pm 10.71$ ) for healthy controls.

The mean disease duration was $21.95 \pm 12.51$ months, the mean Hoehn and Yahr scale was $1.66 \pm 0.48$ and the mean of UPDRS-III was $19.1 \pm 7.2$. Baseline patient demographics and ophthalmologic characteristics are summarized in Table 1.
None of the pRNFL values for the average, temporal (T), supero-temporal (TS), supero-nasal (NS), nasal (N), infero-nasal (NI), infero-temporal (TI), global (G) regions disclosed any significant differences between patients with PD and HC (Table 2).

The Fundus-perimetry examinations were obtained for 33 eyes of 18 patients with PD and 33 eyes of $20 \mathrm{HC}$, considering only examinations with high-quality infrared images.

The statistical analysis of the SD-OCT entire posterior pole area, consisting of 64 values for each retinal layer, revealed a statistically significant decrease in thickness of the outer nuclear layer (ONL) in patients with Parkinson's disease, with a p-value $<0.0001$. On the contrary, a significant increase in thickness of the OPL, with a p-value $<0.0001$, and of the RPE with a p-value $=0.002$ compared to healthy controls were detected. Other retinal layers showed no significant statistical differences (Table 3).

The DLS values measured by MP1 in fovea were significantly lower in patients than in healthy controls (15 [13-16.2] vs 17.95 [16.08-18.96] p-value < 0.0001).

The difference between all the DLS values from the PP MP1 examination pattern in patients was also statistically significant when compared to HC (15.0 [12.0-17.0] vs 18.0 [16.0-20.0] p-value $<0.0001$ ) (Table 4).

In the patient's group, the Pearson correlation between the 64 DLS values of the MP1 pattern, and the 64 corresponding PP thickness values of the OPL, ONL, and RPE 
Table I Demographic and Clinical Information of Patients with Parkinson's Disease (PD) and Healthy Controls (HC)

\begin{tabular}{|l|c|c|c|}
\hline & PD & HC & P value \\
\hline Number of subjects (eyes) & $21(4 I)$ & $57.89 \pm 10.71$ & 0.07 I \\
\hline Age, year & $63 \pm 10.32$ & 12 & $\mathbf{0 . 4 1 6 *}$ \\
\hline $\begin{array}{l}\text { Sex, } \mathrm{n} \\
\text { Male } \\
\text { Female }\end{array}$ & 14 & 10 & \\
\hline H\&Y & 7 & - & \\
\hline UPDRS-III & $1.98 \pm 0.58$ & - & \\
\hline Disease duration, months & $19.1 \pm 7.21$ & - & \\
\hline LEDD & $21.95 \pm 12.51$ & $15.27 \pm 1.49$ & 0.97 \\
\hline Intraocular Pressure, $\mathrm{mmHg}$ & $252.85 \pm 85.74$ & $0.43(0.58)$ & 0.48 \\
\hline Spherical equivalent, D & $15.29 \pm 1.19$ & $0.60(0.90)$ & \\
\hline
\end{tabular}

Notes: P: Statistical difference for ANOVA $(P<0.05)$. Data expressed as means with standard deviation $(S D)$ in case of continuous variables. Values are expressed as mean \pm SD. *Chi-square test.

Abbreviations: H\&Y, Hoehn \& Yahr Stage; UPDRS-III, Unified Parkinson's Disease Rating Scale Part III; LEDD, levodopa equivalent daily dose.

Table 2 pRNFL Thicknesses and Statistical Differences Between Patients with Parkinson's Disease (PD) and Healthy Controls (HC)

\begin{tabular}{|l|c|c|c|}
\hline Thickness $(\boldsymbol{\mu m})$ & PD & HC & P value \\
\hline T & $69.37 \pm 11.14$ & $65.51 \pm 6.99$ & 0.064 \\
TS & $122.56 \pm 23.78$ & $120.10 \pm 21.01$ & 0.621 \\
G & $97.15 \pm 6.91$ & $94.24 \pm 7.62$ & 0.074 \\
NS & $111.66 \pm 29.27$ & $106.10 \pm 15.02$ & 0.282 \\
N & $84.15 \pm 11.48$ & $79.54 \pm 9.99$ & 0.056 \\
NI & $110.37 \pm 25.65$ & $109.34 \pm 21.03$ & 0.844 \\
TI & $148.83 \pm 21.02$ & $143.68 \pm 16.77$ & 0.224 \\
\hline
\end{tabular}

Notes: P: Statistical difference for ANOVA ( $P<0.05)$. Data expressed as means with standard deviation (SD).

Abbreviations: T, thickness of the temporal; TS, supero-temporal; NS, supero-nasal; N, nasal; NI, infero-nasal; TI, infero-temporal; G, global.

Table 3 Thicknesses of Individual Retinal Layers in Retinal Posterior Pole and Statistical Difference Between Patients with Parkinson's Disease (PD) and Healthy Controls (HC)

\begin{tabular}{|l|c|c|c|}
\hline Thickness $(\boldsymbol{\mu m})$ & PD & HC & P value \\
\hline Total Retina & $293.09 \pm 36.17$ & $291.98 \pm 59.53$ & 0.421 \\
PPRNFL & $44.70 \pm 28.59$ & $43.31 \pm 28.27$ & 0.086 \\
GCL & $31.70 \pm 10.54$ & $31.69 \pm 9.99$ & 0.974 \\
IPL & $26.42 \pm 8.82$ & $26.09 \pm 8.40$ & 0.165 \\
INL & $30.73 \pm 6.21$ & $30.60 \pm 5.49$ & 0.43 \\
OPL & $28.78 \pm 6.22$ & $26.64 \pm 4.70$ & $<0.0001$ \\
ONL & $54.25 \pm 11.99$ & $56.17 \pm 12.36$ & $<0.0001$ \\
RPE & $13.36 \pm 2.04$ & $13.19 \pm 1.89$ & 0.002 \\
\hline
\end{tabular}

Notes: P: statistical difference for ANOVA ( $\mathrm{P}<0.05)$. Data expressed as means with standard deviation (SD).

Abbreviations: PPRNFL, posterior pole retinal nerve fiber layer; GCL, ganglion cell layer; IPL, inner plexiform layer; INL, inner nuclear layer; OPL, outer plexiform layer; ONL, outer nuclear layer; RPE, retinal pigmented epithelium. 
Table 4 Difference Between DLS Values Between Patients with Parkinson's Disease (PD) and Healthy Controls (HC)

\begin{tabular}{|l|c|c|c|}
\hline DLS (dB) & PD & HC & P value \\
\hline Foveal & $15[13-16.2]$ & $17.95[16.08-18.96]$ & $<0.0001$ \\
PPole MPI & $15.0[12.0-17.0]$ & $18.0[16.0-20.0]$ & $<0.0001$ \\
\hline
\end{tabular}

Notes: P: statistical difference for Mann-Whitney $(\mathrm{P}<0.05)$. Data expressed as median and $\left[1^{\circ}\right.$ interquartile $-3^{\circ}$ interquartile]

Abbreviations: DLS, differential light sensitivity; PPole MPI, microperimetry matched to OCT posterior pole pattern.

retinal layers was weak, with an $r$ index lower than 0.3 . No significant correlation was found between clinical variables, including the UPDRS III, H\&Y stage, LEDD and individual retinal layers thickness (data not reported).

\section{Discussion}

OCT methodologies have been applied to PD for several years and the overall findings are not univocal and reproducible, as a recent critical review has shown. Such a lack of consistency inevitably leads to caution in interpreting the results of each individual study. ${ }^{16}$

In our work, we used a combined FP and SD-OCT approach to assess "layer-by-layer" retinal thickness and function at retinal PP level in a group of PD patients compared to healthy controls.

Our results showed that the retinal structure in the posterior pole differed statistically between patients with early PD and controls; in particular, in patients' eyes, the outer nuclear layer (ONL) was thinner, whereas the outer plexiform layer (OPL) and the retinal pigmented epithelium (RPE) were thicker.

Two recent reviews and meta-analyses have reported a thinning of the inner retinal layers and the pRNFL as prominent features in the eyes of Parkinson's patients. ${ }^{17,18}$ Other groups also found significant thinning of pRNFL in PD compared to $\mathrm{HC},{ }^{17,19,20}$ however the authors pointed out that their analysis gathered results from patients with different stages of Parkinson's disease. We found no significant difference in the pRNFL thickness between patients with PD and HC (Table 2). Our results are in agreement with those of other Parkinson's studies conducted with similar methods in patients with early-stage Parkinson's disease. ${ }^{21,22}$

Furthermore, Chorostecki et al found a significant increase in OPL thickness in Parkinson's patients in the early stage. The increased thickness of the OPL in our patients is consistent with results from other studies suggesting local accumulation of $\alpha$-syn in this layer, ${ }^{23-25}$ since Parkinson's Disease is characterized by pathological deposits of a-synuclein throughout the central nervous system. However, other authors have shown that phosphorylated $\alpha$-syn accumulates in the retina between the GCL and IPL, in parallel with that in the brain. ${ }^{26}$

Inefficient clearance of $\alpha$-syn via the autophagy-lysosome pathway has long been linked to $\mathrm{PD} ;{ }^{27}$

The inability to degrade $\alpha$-syn increases the cytosolic concentration of this protein by promoting its oligomerization and aggregation. ${ }^{28}$ Pathological accumulation of $\alpha$ syn can compromise several steps of this pathway, ${ }^{29-31}$ thus causing increased levels of oxidized proteins and of potentially toxic intracellular aggregates.

Furthermore, as $\alpha$-syn impairs ferritinophagy, it has an effect on the photoreceptors, which are rich in iron and ferritinoportin.

Photoreceptors place an important metabolic load on RPE cells, due to their high turnover and the management of released iron. Iron is essential for photoreceptors survival, but its homeostatic dysregulation is potentially highly toxic, due to its ability to generate free radicals. ${ }^{32,33}$

Ferritinophagy is fundamental for the iron release from ferritin. Because ferritin must be degraded to release iron, it binds to the lysosome through an autophagosome and is degraded here through hydrolases and releases previously incorporated iron. It is possible that $\alpha$-syn affects ferritinophagy in the RPE, as it blocks the transport of hydrolases to the lysosome and consequently ferritin lysis does not occur. This could explain why we found a thickening of the RPE: ferritin, failing to be degraded in the RPE, accumulates in this layer leading to its thickening. ${ }^{34}$ Since iron metabolism undergoes downregulation at RPE level, it leads to an inhibition of iron transport by ferroportin. As already mentioned above, the accumulation of iron has a toxic effect on tissues, possibly explaining the slight thinning of the ONL (containing the nuclei of photoreceptors) in our study.

Another hypothesis that could explain the thinning of the ONL layer is linked to proteotoxic pathways induced by $\alpha$ - synuclein: mitochondrial dysfunction, endoplasmic reticulum (ER) stress and altered ER-Golgi transport may play relevant roles in this process: the capacity of $\alpha$-synuclein to interact with specific proteins of the cell organelles 
and the cytosol could be crucial factors in the toxicity of this protein.

The reduction of the DLS that we measured by FP may be related to the well-known alteration of contrast sensitivity in Parkinson's patients; ${ }^{35-37}$ this may stem from dopaminergic denervation of retinal amacrine cells. ${ }^{38}$

The first clinical reports of abnormal contrast sensitivity in Parkinson's disease came from Regan and Neima ${ }^{39}$ in 1984, when they studied the visual function of 10 patients using letter charts similar to Snellen cards, but with varying levels of contrast. Autopsy case studies in PD revealed loss of dopaminergic innervation around the fovea and decreased retinal dopamine concentration despite the preservation of retinal dopaminergic neurons. ${ }^{40,41}$ Dopamine is essential for light adaptation, as it facilitates the transmission of visual signals in rod and cone circuits and exerts multiple trophic effects on retinal cells. FP explores the DLS thresholds in mesopic adaptation due to the low luminance level (4 apostilbs) of its background. This might have contributed to revealing central DLS deficits in our patients even though most of them were in the early stage. Furthermore, previous studies with standard automatic perimetry had already shown a reduction in differential light sensitivity in patients with Parkinson's disease even in presence of a normal retinal structure detected by OCT. ${ }^{42,43}$

Some electrophysiological studies in the literature on patients with Parkinson's disease demonstrated delayed implicit times and decreased amplitude responses in the visual evoked potentials and pattern electroretinogram in PD subjects ${ }^{44}$ as well as multifocal electroretinography (mfERG) changes in patients with PD without visual loss. $^{45}$

Husman et al compared the FP to the mfERG in hydroxychlororoquine maculopathy ${ }^{46}$ concluding that the detection frequency of hydroxychloroquine toxicity with FP was greater than with mfERG. FP showed good sensitivity and moderate specificity in detecting functional deficits induced by hydroxychlororoquine. Santos et al compared the results between mfERG and FP in diabetic macular edema and concluded that both were able to demonstrate a functional improvement after intravitreal treatment with ranibizumab. ${ }^{47}$ In general, fundus perimetry seems to perform as well as electrophysiology examination in detecting retinal alterations in different pathologies.

Our study showed for the first time a decrease in DLS in the whole retinal posterior pole using FP examination. This is in agreement with results reported by Han et $\mathrm{al}^{48}$ using polar three test pattern of SD-OCT OPKO OTI microperimetry in the ETDRS area and with similar changes reported using standard automated perimetry (SAP) or pattern electroretinography (pERG) or mfERG in early Parkinson's disease patients.

Using the fundus perimetry/OCT overlay method, ${ }^{9}$ we found that morphological and functional results did not tend to vary together. The low correlation coefficients found between FP DLS values and OCT thickness are not surprising since neither the ganglion cell nor the retinal nerve fiber layers showed significant alterations in our patients. However, the low correlation can also be partly explained by the failure to detect small scotomas due to the upper truncation of the extension of the MP1 DLS exploration range. ${ }^{49}$

A further aspect to be considered is that FP is a fairly long-duration psychophysical examination that requires constant attention, possibly challenging for patients. Other limits of the study were the relatively small sample size and the cross-sectional and case-control design.

\section{Conclusion}

In conclusion, in our study, fundus perimetry DLS defects were measured with accurate, layer by layer, topographical correspondence with the SD-OCT volumetric units in the entire retinal posterior pole in patients with early Parkinson's Disease.

Our results are very similar to those of Chorostecki et al who also found a thickening of OPL using the same SD-OCT as the identical segmentation tool but in the macular area. Their patients had a disease duration of 6.4 \pm 5.1 years and our patients of $21.95 \pm 12.51$ months. Thus, it seems reasonable that OPL thickening could represent an OCT feature of the early stages of Parkinson's disease, probably due to initial alpha-synuclein accumulation; as the disease progresses, toxic $\alpha$ synuclein aggregates could lead to a consistent loss of ganglion cells and their axons causing a thinning of inner retinal layers, mostly described by other Authors in the eyes of Parkinson patients. Only multicentric, prospective, long-term, randomized clinical trials will be able to clarify the real nature and time course of retinal damage in Parkinson's patients.

\section{Disclosure}

The author reports no conflicts of interest in this work. 


\section{References}

1. Williams DR, Litvan I. Parkinsonian syndromes. Continuum. 2013;19 (5Movement Disorders):1189-1212.

2. Parnetti L, Castrioto A, Chiasserini D, et al. Cerebrospinal fluid biomarkers in Parkinson disease. Nat Rev Neurol. 2013;9(3):131140. doi: $10.1038 /$ nrneurol.2013.10

3. Michell AW, Lewis SJ, Foltynie T, Barker RA. Biomarkers and Parkinson's disease. Brain. 2004;127(Pt 8):1693-1705. doi:10.1093/ brain/awh198

4. Kalia LV, Lang AE. Parkinson's disease. Lancet. 2015;386 (9996):896-912. doi:10.1016/S0140-6736(14)61393-3

5. Armstrong MJ, Okun MS. Diagnosis and treatment of Parkinson disease: a review. JAMA. 2020;323(6):548-560. doi:10.1001/ jama.2019.22360

6. Delenclos M, Jones DR, McLean PJ, Uitti RJ. Biomarkers in Parkinson's disease: advances and strategies. Parkinsonism Relat Disord. 2016;22(Suppl 1):S106-110. doi:10.1016/j.parkreldis.2015. 09.048

7. Guo L, Normando EM, Shah PA, De Groef L, Cordeiro MF. Oculovisual abnormalities in Parkinson's disease: possible value as biomarkers. Mov Disord. 2018;33(9):1390-1406. doi:10.1002/mds.27454

8. Lad EM, Mukherjee D, Stinnett SS, et al. Evaluation of inner retinal layers as biomarkers in mild cognitive impairment to moderate Alzheimer's disease. PLoS One. 2018;13(2):e0192646. doi:10.1371/ journal.pone. 0192646

9. Cesareo M, Ciuffoletti E, Martucci A, et al. Assessment of the retinal posterior pole in dominant optic atrophy by spectral-domain optical coherence tomography and microperimetry. PLoS One. 2017;12(3): e0174560. doi:10.1371/journal.pone.0174560

10. Pillai JA, Bermel R, Bonner-Jackson A, et al. Retinal Nerve Fiber Layer Thinning in Alzheimer's Disease: a Case-Control Study in Comparison to Normal Aging, Parkinson's Disease, and NonAlzheimer's Dementia. Am $J$ Alzheimers Dis Other Demen. 2016;31(5):430-436. doi:10.1177/1533317515628053

11. Satue M, Garcia-Martin E, Fuertes I, et al. Use of Fourier-domain OCT to detect retinal nerve fiber layer degeneration in Parkinson's disease patients. Eye. 2013;27(4):507-514. doi:10.1038/eye.2013.4

12. Moreno-Ramos T, Benito-Leon J, Villarejo A, Bermejo-Pareja F. Retinal nerve fiber layer thinning in dementia associated with Parkinson's disease, dementia with Lewy bodies, and Alzheimer's disease. J Alzheimers Dis. 2013;34(3):659-664. doi:10.3233/JAD121975

13. Moschos MM, Chatziralli IP. Evaluation of choroidal and retinal thickness changes in parkinson's disease using spectral domain optical coherence tomography. Semin Ophthalmol. 2018;33(4):494-497. doi: $10.1080 / 08820538.2017 .1307423$

14. Ma LJ, Xu LL, Mao CJ, et al. Progressive Changes in the Retinal Structure of Patients with Parkinson's Disease. J Parkinsons Dis. 2018;8(1):85-92. doi:10.3233/JPD-171184

15. Postuma RB, Berg D, Stern M, et al. MDS clinical diagnostic criteria for Parkinson's disease. Mov Disord. 2015;30(12):1591-1601. doi: $10.1002 / \mathrm{mds} .26424$

16. Mailankody P, Lenka A, Pal PK. The role of optical coherence tomography in parkinsonism: a critical review. J Neurol Sci. 2019;403:67-74. doi:10.1016/j.jns.2019.06.009

17. Huang L, Wang C, Wang W, Wang Y, Zhang R. The specific pattern of retinal nerve fiber layer thinning in Parkinson's disease: a systematic review and meta-analysis. $J$ Neurol. 2020;20:1-10.

18. Huang L, Zhang D, Ji J, Wang Y, Zhang R. Central retina changes in Parkinson's disease: a systematic review and meta-analysis. J Neurol. 2020;10:1-9.

19. Garcia-Martin E, Satue M, Fuertes I, et al. Ability and reproducibility of Fourier-domain optical coherence tomography to detect retinal nerve fiber layer atrophy in Parkinson's disease. Ophthalmology. 2012;119(10):2161-2167. doi:10.1016/j.ophtha.2012.05.003
20. Rohani M, Langroodi AS, Ghourchian S, Falavarjani KG, SoUdi R, Shahidi G. Retinal nerve changes in patients with tremor dominant and akinetic rigid Parkinson's disease. Neurol Sci. 2013;34(5):689693. doi:10.1007/s10072-012-1125-7

21. Aaker GD, Myung JS, Ehrlich JR, Mohammed M, Henchcliffe C, Kiss S. Detection of retinal changes in Parkinson's disease with spectral-domain optical coherence tomography. Clin Ophthalmol. 2010;4:1427-1432.

22. Albrecht P, Muller AK, Sudmeyer M, et al. Optical coherence tomography in parkinsonian syndromes. PLoS One. 2012;7(4):e34891. doi:10.1371/journal.pone.0034891

23. Chorostecki J, Seraji-Bozorgzad N, Shah A, et al. Characterization of retinal architecture in Parkinson's disease. J Neurol Sci. 2015;355(12):44-48. doi:10.1016/j.jns.2015.05.007

24. Unlu M, Gulmez Sevim D, Gultekin M, Karaca C. Correlations among multifocal electroretinography and optical coherence tomography findings in patients with Parkinson's disease. Neurol Sci. 2018;39(3):533-541. doi:10.1007/s10072-018-3244-2

25. Martinez-Navarrete GC, Martin-Nieto J, Esteve-Rudd J, Angulo A, Cuenca N. Alpha synuclein gene expression profile in the retina of vertebrates. Mol Vis. 2007;13:949-961.

26. Ortuno-Lizaran I, Beach TG, Serrano GE, Walker DG, Adler CH, Cuenca N. Phosphorylated alpha-synuclein in the retina is a biomarker of Parkinson's disease pathology severity. Mov Disord. 2018;33 (8):1315-1324. doi:10.1002/mds.27392

27. Anglade P, Vyas S, Javoy-Agid F, et al. Apoptosis and autophagy in nigral neurons of patients with Parkinson's disease. Histol Histopathol. 1997;12(1):25-31.

28. Rideout HJ, Lang-Rollin I, Stefanis L. Involvement of macroautophagy in the dissolution of neuronal inclusions. Int J Biochem Cell Biol. 2004;36(12):2551-2562. doi:10.1016/j.biocel.2004.05.008

29. Xilouri M, Brekk OR, Stefanis L. Autophagy and alpha-synuclein: relevance to Parkinson's disease and related synucleopathies. Mov Disord. 2016;31(2):178-192. doi:10.1002/mds.26477

30. Markossian KA, Kurganov BI. Protein folding, misfolding, and aggregation. Formation of inclusion bodies and aggresomes. Biochemistry (Mosc). 2004;69(9):971-984. doi:10.1023/B: BIRY.0000043539.07961.4c

31. Cuervo AM, Stefanis L, Fredenburg R, Lansbury PT, Sulzer D. Impaired degradation of mutant alpha-synuclein by chaperonemediated autophagy. Science. 2004;305(5688):1292-1295. doi:10.1126/science. 1101738

32. Ben-Shachar D, Riederer P, Youdim MB. Iron-melanin interaction and lipid peroxidation: implications for Parkinson's disease. $J$ Neurochem. 1991;57(5):1609-1614. doi:10.1111/j.1471-4159.1991. tb06358.x

33. Shu W, Dunaief JL. Potential treatment of retinal diseases with iron chelators. Pharmaceuticals. 2018;11(4). doi:10.3390/ph11040112

34. Baksi S, Singh N. alpha-Synuclein impairs ferritinophagy in the retinal pigment epithelium: implications for retinal iron dyshomeostasis in Parkinson's disease. Sci Rep. 2017;7(1):12843. doi:10.1038/ s41598-017-12862-x

35. Ming W, Palidis DJ, Spering M, McKeown MJ. Visual contrast sensitivity in early-stage Parkinson's disease. Invest Ophthalmol Vis Sci. 2016;57(13):5696-5704. doi:10.1167/iovs.16-20025

36. Satue M, Rodrigo MJ, Obis J, et al. Evaluation of Progressive Visual Dysfunction and Retinal Degeneration in Patients With Parkinson's Disease. Invest Ophthalmol Vis Sci. 2017;58(2):1151-1157. doi:10.1167/iovs. 16-20460

37. Uc EY, Rizzo M, Anderson SW, Qian S, Rodnitzky RL, Dawson JD. Visual dysfunction in Parkinson disease without dementia. Neurology. 2005;65(12):1907-1913. doi:10.1212/01.wnl.00001915 65.11065.11

38. Ortuno-Lizaran I, Sanchez-Saez X, Lax P, et al. Dopaminergic retinal cell loss and visual dysfunction in Parkinson disease. Ann Neurol. 2020;88(5):893-906. doi:10.1002/ana.25897 
39. Regan D, Neima D. Low-contrast letter charts in early diabetic retinopathy, ocular hypertension, glaucoma, and Parkinson's disease. Br J Ophthalmol. 1984;68(12):885-889. doi:10.1136/bjo.68.12.885

40. Harnois C, Di Paolo T. Decreased dopamine in the retinas of patients with Parkinson's disease. Invest Ophthalmol Vis Sci. 1990;31 (11):2473-2475.

41. Nguyen-Legros J. Functional neuroarchitecture of the retina: hypothesis on the dysfunction of retinal dopaminergic circuitry in Parkinson's disease. Surg Radiol Anat. 1988;10(2):137-144. doi:10.1007/BF02307822

42. Tsironi EE, Dastiridou A, Katsanos A, et al. Perimetric and retinal nerve fiber layer findings in patients with Parkinson's disease. $B M C$ Ophthalmol. 2012;12:54. doi:10.1186/1471-2415-12-54

43. Yenice O, Onal S, Midi I, Ozcan E, Temel A, Ig D. Visual field analysis in patients with Parkinson's disease. Parkinsonism Relat Disord. 2008;14(3):193-198. doi:10.1016/j.parkreldis.2007.07.018

44. Garcia-Martin E, Rodriguez-Mena D, Satue M, et al. Electrophysiology and optical coherence tomography to evaluate Parkinson disease severity. Invest Ophthalmol Vis Sci. 2014;55 (2):696-705. doi:10.1167/iovs.13-13062
45. Moschos MM, Tagaris G, Markopoulos I, et al. Morphologic changes and functional retinal impairment in patients with Parkinson disease without visual loss. Eur $J$ Ophthalmol. 2011;21(1):24-29. doi:10.5301/EJO.2010.1318

46. Husman A, Padhi TR, Chen A, et al. Comparison of fundus-guided microperimetry and multifocal electroretinography for evaluating hydroxychloroquine maculopathy. Transl Vis Sci Technol. 2019;8 (5):19. doi:10.1167/tvst.8.5.19

47. Santos AR, Raimundo M, Alves D, et al. Microperimetry and mfERG as functional measurements in diabetic macular oedema undergoing intravitreal ranibizumab treatment. Eye. 2021;35(5):1384-1392. doi:10.1038/s41433-020-1054-2

48. Han J, Lee JY, Kim TW, et al. Retinal thinning associates with nigral dopaminergic loss in de novo Parkinson disease. Neurology. 2018;91 (11):e1003-e1012. doi:10.1212/WNL.0000000000006157

49. Midena E, Vujosevic S, Cavarzeran F; Microperimetry Study G. Normal values for fundus perimetry with the microperimeter MP1. Ophthalmology. 2010;117(8):1571-1576, 1576 e1571. doi:10.1016/j. ophtha.2009.12.044
Clinical Ophthalmology

\section{Publish your work in this journal}

Clinical Ophthalmology is an international, peer-reviewed journal covering all subspecialties within ophthalmology. Key topics include: Optometry; Visual science; Pharmacology and drug therapy in eye diseases; Basic Sciences; Primary and Secondary eye care; Patient Safety and Quality of Care Improvements. This journal is indexed on PubMed

\section{Dovepress}

Central and CAS, and is the official journal of The Society of Clinical Ophthalmology (SCO). The manuscript management system is completely online and includes a very quick and fair peer-review system, which is all easy to use. Visit http://www.dovepress.com/ testimonials.php to read real quotes from published authors. 